\title{
Microinvasive Mitral Valve Surgery: A New Frontier to Tackle Mitral Regurgitation
}

\author{
Solomon Seifu ${ }^{1}$ and Eduardo de Marchena ${ }^{1}$ \\ ${ }^{1}$ University of Miami School of Medicine
}

September 24, 2021

\begin{abstract}
Microinvasive, catheter-based mitral valve repair of severe mitral regurgitation utilizes less invasive approaches with less procedural morbidity and mortality. The procedural steps and clinical benefits of the transcatheter transapical mitral valve annuloplasty (AMEND mitral repair implant) and transcatheter transapical chordal repair systems (Neochord DS 1000 device and Harpoon Mitral Valve Repair System) are reviewed in this manuscript.
\end{abstract}

Solomon Seifu, MD

Eberhardt Grube Interventional Structural heart disease Fellow 2021-2022

Division of Cardiovascular Medicine

University of Miami/Jackson Memorial Hospital

Office Phone number: 305-243-9200

1400 NW 10 ${ }^{\text {th }}$ Avenue Dominion Tower Suite 206A Miami Fl. 33136

Corresponding Author:

Eduardo de Marchena MD, FACC, FACP, FSCAI

Associate Dean for International Medicine

Professor of Medicine \& Surgery

Director, Interventional Cardiology

Program Director, Eberhard Grube International Structural Heart Training Program

University of Miami Miller School of Medicine, University of Miami

Office Phone number 305-243-9200

1400 NW 10 ${ }^{\text {th }}$ Avenue Dominion Tower Suite 206A Miami Fl. 33136

Microinvasive Mitral Valve Surgery: A New Frontier to Tackle Mitral Regurgitation

Solomon Seifu, MD; Eduardo de Marchena, MD

Mitral regurgitation can be caused by either primary mitral valve apparatus disease process which is called primary MR/degenerative mitral valve disease or secondary causes from ventricular remodeling which is called functional MR. The mitral valve apparatus includes the valve leaflets, annulus, chordae tendineae, and papillary muscles. The left atrium and ventricle are also integrally involved with mitral valve function. 
Treatment of mitral valve regurgitation therefore should primarily focus on the specific pathophysiologic process leading to the MR.

Treatment of mitral regurgitation has evolved from an open-heart surgical repair to microinvasive surgical repair and transcatheter repair. Surgical mitral valve repair techniques can involve a simple edge-to-edge repair using an Alfieri's stitch or use adjunctive artificial chord replacement and ring annuloplasty. Catheterbased mitral valve repair utilizes less invasive approaches with less procedural morbidity and mortality. Percutaneous annuloplasty can be achieved indirectly via the coronary sinus or directly from retrograde left ventricular access. Catheter-based leaflet repair is accomplished using an implantable clip to mimic the surgical edge-to-edge technique [1].

In this issue of Journal of Cardiac surgery, D'Onofrio et al [2], the authors review the technologies intended to treat mitral regurgitation (MR) through transapical approach, including annuloplasty and chordal-repair options. They reviewed the procedural steps and clinical results of the transcatheter transapical mitral valve annuloplasty (AMEND mitral repair implant) and transcatheter transapical chordal repair systems (Neochord DS 1000 device and Harpoon Mitral Valve Repair System).

The AMEND (Valcare Medical, Herzliya Pituach, Israel) is a transcatheter device for direct mitral annuloplasty. It is a D-shaped ring with anchoring capabilities that mimics the annuloplasty rings used in open-heart surgery and allows for anterior-posterior reduction. The procedure is performed under general anesthesia through transapical approach with the guidance of transesophageal echocardiography and fluoroscopy. Implantations performed in a mix of 14 functional and degenerative MR patients showed reduction of MR to [?] 2+. A $20 \%$ mean reduction of anteroposterior diameter was achieved, no residual pulmonary flow reversal in all cases was reported, mean reduction of the jet area was $74 \%$.

The transcatheter chordal repair technologies reviewed in this manuscript are primarily intended to treat degenerative mitral regurgitations. The Neochord DS 1000 device (Neochord Inc, St. Louis Park, MN) is a transapical beating heart mitral valve repair system with a neochord implantation. The procedure is performed under general anesthesia through the left ventricular apical access with real time transesophageal guidance. The TACT Trial (Transapical Artificial Chordae Tendinae) showed for the first time that the NeoChord DS1000 system is technically safe and feasible [3]. The acute procedural success was reduction of MR to [?] 2+ in $86.7 \%$ of patients. The second transcatheter chordal device discussed is the Harpoon Mitral Valve Repair System (Edwards Lifesciences, Irvine, CA). It is performed under general anesthesia and through transapical access with real time transesophageal echocardiography guidance. The TRACER trial (Mitral TransApicalNeoChordal Echo-Guided Repair) demonstrated the safety and effectiveness of this device with MR reduction to moderate or less, met in $90 \%$ of patients [4].

The authors should be applauded for the excellent review of these three innovative technologies of annuloplasty and chordal repair targeting the different components of the mitral valvular apparatus. Transcatheter mitral valve repair technologies are becoming more and more refined in recent years demonstrating a promising clinical success for the treatment of severe mitral regurgitation.

References:

1. Feldman T, Cilingiroglu M. Percutaneous leaflet repair and annuloplasty for mitral regurgitation. J Am Coll Cardiol. 2011;57(5):529-537.

2. D'Onofrio, Augusto, et al. "Transapical mitral valve repair procedures: primetime for microinvasive mitral valve surgery." Authorea Preprints (2021).

3. Seeburger J, Rinaldi M, Nielsen SL, et al. Off-pump transapical implantation of artificial neo-chordae to correct mitral regurgitation: the TACT Trial (Transapical Artificial Chordae Tendinae) proof of concept. J Am Coll Cardiol. 2014;63(9):914-919.

4. Gammie JS, Bartus K, Gackowski A, et al. Beating-Heart Mitral Valve Repair Using a Novel ePTFE Cordal Implantation Device: A Prospective Trial. J Am Coll Cardiol. 2018;71(1):25-36. 